\title{
A study of sine-wave contrast sensitivity by two psychophysical methods*
}

\author{
D. H. KELLY and R. E. SAVOIE \\ Stanford Research Institute, Menlo Park, California 94025
}

\begin{abstract}
In the literature on visual contrast thresholds for sine wave gratings, little attention has been paid to the psychophysical methods used to obtain these spatial-frequency response curves. Here we report a comparison of such data obtained by two quite different psychophysical methods, but otherwise under identical conditions, using five Ss. Both experiments were run by computer: (1) In the method of adjustments, the computer program merely controls the order of the stimuli and records S's contrast settings. (2) In the forced-choice staircase (FCS) technique, the program determines how often $S$ can discriminate the sinusoidal grating from a uniform field, informs $S$ of his accuracy, controls the stimulus contrast on the basis of S's preceding responses, and brackets his threshold by a series of successive approximations. Method 2 eliminates criterion effects that occur in Method 1, and hence tends to minimize individual differences. However, the FCS technique requires an order of magnitude more observing time to obtain equally smooth contrast sensitivity curves. FCS also increases the overall sensitivity of some Ss by as much as five times, but it does not significantly change the shape of the contrast sensitivity curve; both methods show strong effects of lateral inhibition at low spatial frequencies.
\end{abstract}

Measurements of the contrast threshold for a sinusoidal grating as a function of its spatial frequency have been used to study the effects on the visual process of optical, neural, chromatic, temporal, and other factors. Van Meeteren (1966) has reviewed a number of these studies. We are particularly interested in the low-frequency region of such data, below about 2 cycles/deg (cpd), because the monotonic increase of contrast sensitivity with increasing spatial frequency in this region may represent a simple form of lateral inhibition (Kelly, 1973).

In certain cases, little or no low-frequency falloff was reported (e.g., Westheimer, 1960; Campbell \& Green, $1965)$, but this has been attributed to the use of small, sharp-edged targets (Davidson, 1966; Kelly, 1970) or flash presentations (Kelly, 1971, 1973), which are unsuitable for isolating the steady-state response to very low spatial frequencies. However, some Ss report that the task of detecting a low-frequency grating seems different from the high-frequency detection task; this raises the question of whether the apparent inhibition would persist at low frequencies if criterion effects were eliminated.

Many of the data in the literature have been obtained by the psychophysical method of adjustments, which is the easiest and fastest procedure when $S s$ are experienced in this type of judgment; variations of the method of limits have also been used. But more sophisticated psychophysical methods have been developed in recent years which are essentially independent of threshold criterion; these have not been

*Our work was partly supported by NIH Grant No. NS-08322 and NSF Grant No. GB-11571. Some of the data were reported at the October 1971 meeting of the Optical Society of America in Ottawa, Canada. We thank T. N. Cornsweet for suggesting this study. applied to the measurement of sine wave contrast thresholds. We therefore undertook to compare the sine wave thresholds obtained by the method of adjustments with those obtained by a forced-choice staircase (FCS) paradigm, in which the $\mathrm{S}$ was always informed of the correctness of his choice. Our main purpose was to find out whether the low-frequency inhibition was independent of criterion effects, but our results are also relevant to other sine wave contrast experiments that use subjective judgments.

\section{METHODS}

The stimulating apparatus is described in detail elsewhere (Kelly, 1966, 1972); its components are shown schematically in Fig. $1 . \mathrm{S}$ is seated comfortably, viewing a cathode ray tube (CRT) $50 \mathrm{~cm}$ distant through an artificial pupil, $2.3 \mathrm{~mm}$ in diam. He sees an 8-deg circular field, filled by a vertical sinusoidal grating. The spatial frequency of this grating is controlled by a (LINC 8) computer. The dependent variable is the Michelson contrast ( $\mathrm{m})$ of the grating, defined as

$$
\mathrm{m}=\frac{\mathrm{B}_{\max }-\mathrm{B}_{\min }}{\mathrm{B}_{\max }+\mathrm{B}_{\min }}
$$

where $B_{\max }$ and $B_{\min }$ are the maximum and minimum values of the stimulus waveform, in trolands (td) of retinal illuminance. This contrast is under the control of either the computer or the $S$, depending on the mode of operation. Since the grating does not flash or flicker in the present experiments, our temporal waveform source was not used (see Fig. 1).

\section{Adjustments Mode}

In the method of adjustments, the $S$ controls the contrast of the grating, using a geared-down potentiometer without stops or other mechanical cues. Spinning the knob about 1,200 deg covers the entire adjustment range, which may be either $0-1$ or $0-0.1$ contrast, depending on a switch controlled by the $S$. Another switch gives him the option of viewing zero or full contrast at any time, without losing his potentiometer setting. When the setting meets his threshold criterion, he pushes a 


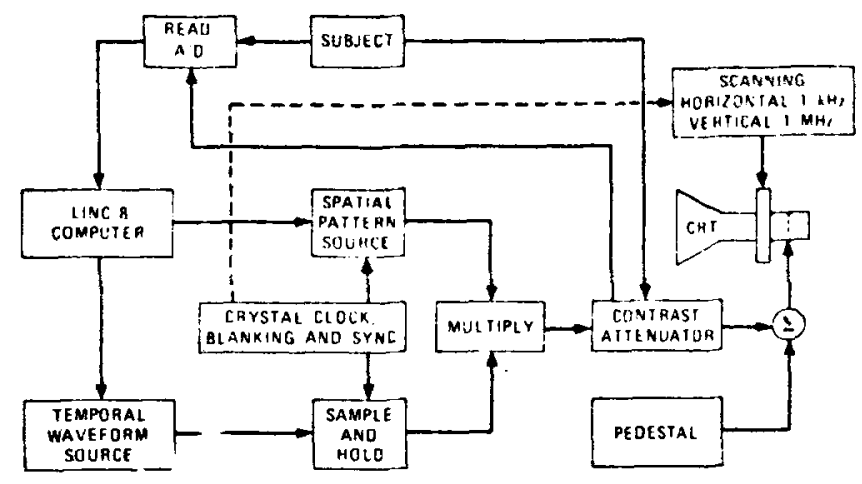

(a) METMOO OF ADJUSTMENT

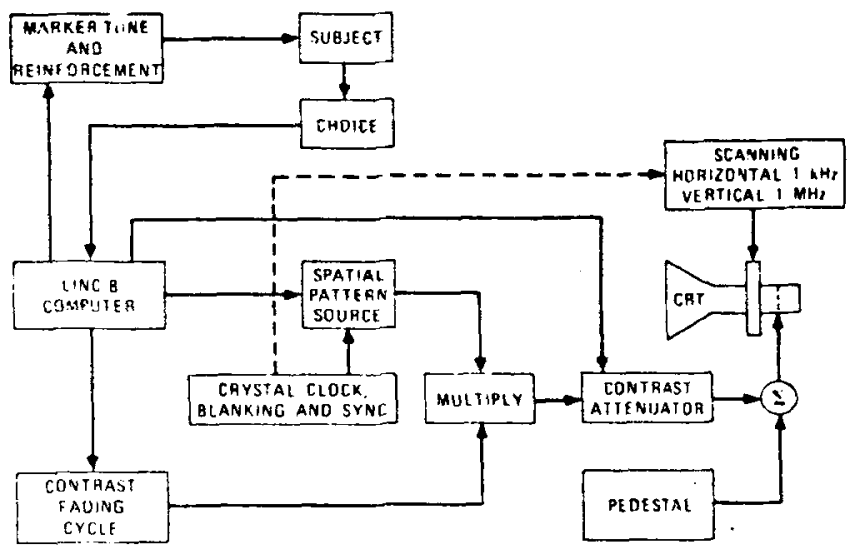

(b) FoACEU.CHOICE STAIRCASE METHOD

Fig. 1. Signal-flow diagrams of the CRT stimulator. (a) Configuration for method of adjustments. (b) Configuration for forced-choice staircase (FCS) procedure.

button which enters this contrast in to the computer.

$\mathrm{He}$ is instructed to fixate the center of the grating and to find the contrast at which it can just be discriminated from a uniform field. (This criterion, which we always use for sine wave thresholds, should come close to matching S's performance in the forced-choice task described below.) $S$ spends as much time as he wishes "hunting" back and forth to find his threshold, but we instruct him to make his final judgments only in the steady state; i.e., after he has refrained from changing the contrast for several seconds. Twelve spatial frequencies were tested in one experimental session; each pattern was presented five times in random order, for a total of 60 judgments. The means of these five settings gave fairly smooth spatial-frequency response curves, as described below. An experienced $S$ can complete such a run in $20-25 \mathrm{~min}$.

\section{Forced-Choice Staircase (FCS) Mode}

Our criterionless psychophysical procedure combined the staircase method of ordering stimuli (Békésy, 1947; Cornsweet, 1962) with the forced-choice method of response collection (Blackwell, 1946; Heinemann, 1961). In this application, the two techniques complement each other in such a way that $S$ spends most of his time making discriminations near threshold.

The S's task is much simpler in the FCS mode. The same 12 stimulus patterns are used, but their contrast is now under the control of the computer. One stimulus cycle consists of two successive intervals, each $5 \mathrm{sec}$ long; a pattern of nonzero contrast is presented in only one of these intervals, which is determined by the computer from a table of random numbers.
One second after the beginning of each interval, a marker tone sounds to notify $S$ that the stimulus may be visible. He makes his choice of interval by pushing a button. which also starts the next trial; but this button has no effect until after the second tone. In other words, $S$ must make a choice in order to start a new trial. but he cannot do so until after the second interval has started. As soon as he makes his choice, he hears a pleasant tone (different from the marker tone) if he is correct or an unpleasant noise if he is not.

His only other control is a "pause" switch, which interrupts the experiment for rest periods. $S$ may use this switch also to abort a given trial (if, for example, he happened to be looking away when the marker tone sounded). The aborted trial is repeated when the pause switch is reset, but the stimulus will not necessarily occur in the same interval.

In order to avoid transient effects (Kelly, 1971, 1973), as we do in the adjustments mode, the temporal waveform of the FCS stimulus is carefully controlled. The mean luminance of the CRT screen is held constant throughout the experiment. When a given pattern is presented, its contrast is smoothly "faded in" from zero to whatever value is set by the computer, as shown in Fig. 2a. The temporal envelope of the fade-in waveform resembles a half-cycle of a $0.5-\mathrm{Hz}$ cosine wave, so that the full contrast of the pattern is present after $1 \mathrm{sec}$ (this contrast always being zero in either the first or second interval, at random). The pattern is also faded out the same way.

All 12 staircases are independent and randomly interleaved, which helps to eliminate subjective bias effects (Cornsweet, 1962). On each trial, the contrast of the FCS stimulus is contingent on the correctness of the preceding responses to the same pattern, according to an algorithm described in detail in the Appendix. When the responses indicate that $S$ can detect the pattern, the contrast is decreased; when he cannot, the contrast is increased, by a constant logarithmic increment in either case. Thus, the contrast is forced to cross and recross the threshold level (which is about $75 \%$ correct). At first, the size of the increment is decreased each time the response sequence indicates that a contrast increase has probably crossed S's threshold. But the third time this indication occurs, the smallest increment (30\% contrast change) is maintained and the staircase is terminated with a fixed number of additional trials.

A typical staircase illustrating these properties is shown in Fig. 2b. Each stimulus is first presented at full contrast, to familiarize $S$ with its appearance and to provide a suprathreshold baseline. If he detects it correctly, its next presentation is at a contrast of 0.02 ; the staircase then enters the main algorithm (see Appendix).

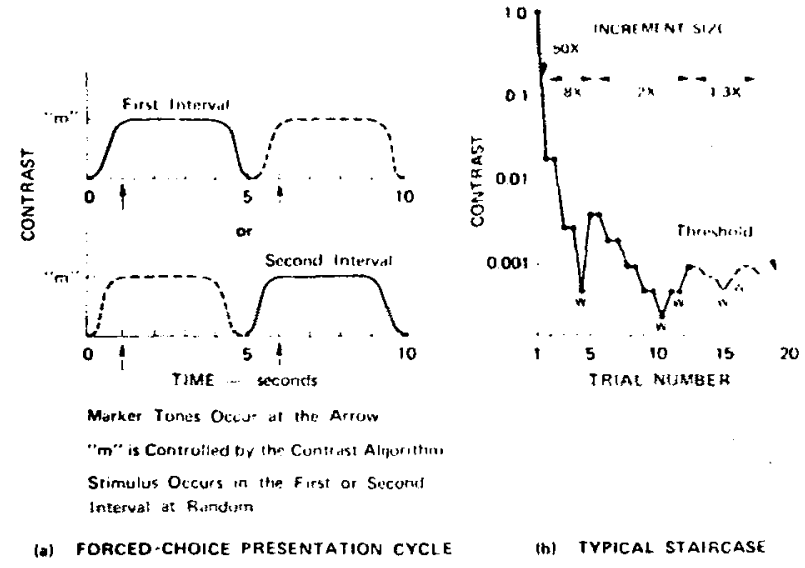

Fig. 2. Details of the FCS procedure. (a) Temporal waveform of one stimulus cycle and timing of alerting tones. (b) A single staircase, reassembled to show all contrasts presented, increment sizes, wrong responses, and calculated threshold. 
Several hundred trials are required to terminate all 12 staircases; an experimental session usually takes $65-75 \mathrm{~min}$, regardless of the skill or experience of the $S$. Most of the variability in length of the individual staircases (Rose et al, 1970) is averaged out by the large number of staircases being run simultaneously. 1

The threshold for each pattern is calculated as the mean of the last eight contrasts presented, no adjacent pair of these being separated by more than the smallest increment. The intersession variability of FCS data obtained in this way is no greater than in the adjustment mode, but is mainly random rather than systematic. Thus, to obtain equally smooth spatial-frequency response curves, one must average the data from three FCS sessions, as described below.

\section{RESULTS}

Figure 3 shows some contrast sensitivity data obtained with a 20 -year-old emmetropic naive $\mathrm{S}$. The upper curve (filled circles) represents combined data from three FCS runs; three comparable adjustment curves are plotted separately (open circles). These data are typical of our results in three ways. First, the shape of the FCS curve is essentially the same as that of the adjustment curves; in both cases, the contrast sensitivity increases steeply at low spatial frequencies, to a maximum near $4 \mathrm{cpd}$. Second, the FCS sensitivity is systematically greater than that in any of the adjustment runs, by a factor of 2 to 5 . Third, there are also systematic differences among the individual adjustment curves, but these are smaller than the FCS-adjustment differences.

The lowest sensitivity in Fig. 3 represents the first

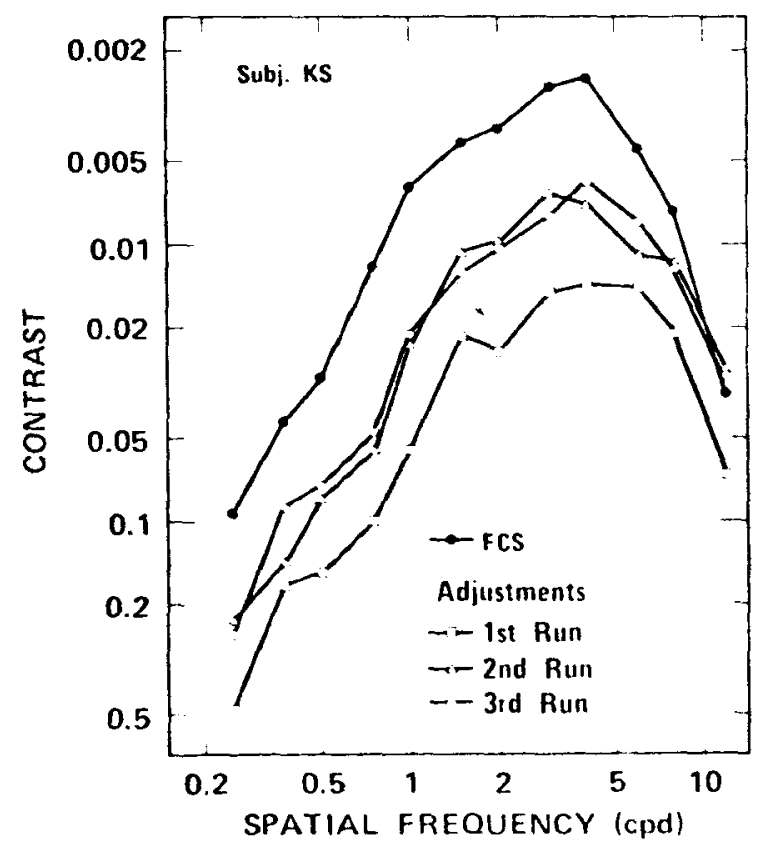

Fig. 3. Contrast sensitivity vs spatial frequency for S K.S. Retinal illuminance, $1,300 \mathrm{td}$; artificial pupil, 2. mm. Filled circles are the means of three FCS runs. Open circles represent three successive adjustment runs.

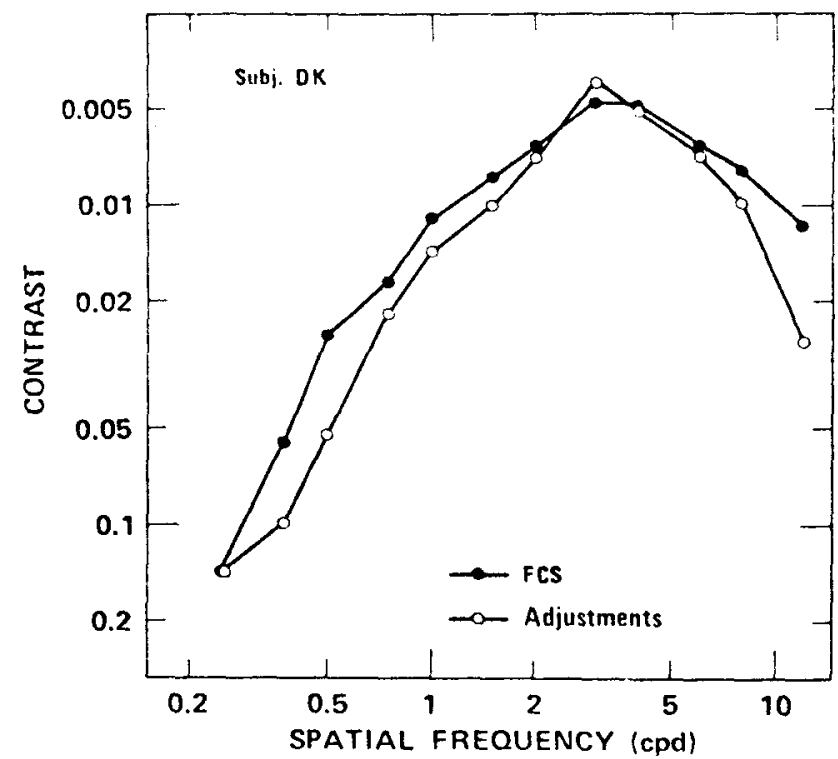

Fig. 4. Contrast sensitivity vs spatial frequency for $S$ D.K. Same conditions as Fig. 3.

experiment with SK.S. Her adjustment sensitivity increased subsequently, but leveled off between the second and third adjustment runs. Typically, a naive $S$ starts with a high threshold criterion, which he then lowers after some experience with the method of adjustments. However, his adjustment thresholds seldom get as low as his FCS thresholds; apparently he adopts a criterion in the range of $90 \%-100 \%$ probability of detection (compared to the $75 \%$ imposed by the FCS mode).

Figure 4 shows a similar comparison for another $\mathrm{S}$. S D.K. (one of the authors) is somewhat atypical, having had hundreds of hours of practice in experiments with this particular apparatus. If practice lowers the adjustment threshold, his adjustment sensitivity should be greater than that of S K.S.; and it is, relative to his FCS sensitivity. His adjustment sensitivity may still be slightly less than his FCS sensitivity, but the two are much closer together than they are for our other Ss. Evidently he uses a lower threshold criterion, closer to the FCS level. As in Fig. 3, the FCS curve in Fig. 4 was obtained by averaging data from three sessions, a total of about $3.5 \mathrm{~h}$ observing time.

However, the contrast sensitivity curves obtained from individual FCS sessions are also instructive; these are shown for a third $\mathrm{S}$ in Fig. 5. Each of the dashed curves in this figure connects the end points of the 12 staircases obtained in a single experimental session (see Fig. 2b). The mean curve for these three FCS sessions is shown for comparison with the previous figures. Figure 5 also shows some systematic variation among three adjustment runs for this S. (S H.P. was more experienced than K.S., but even an experienced $S$ will not always hold the same adjustment criterion from one run to the next.) Again, the FCS sensitivity is about five 


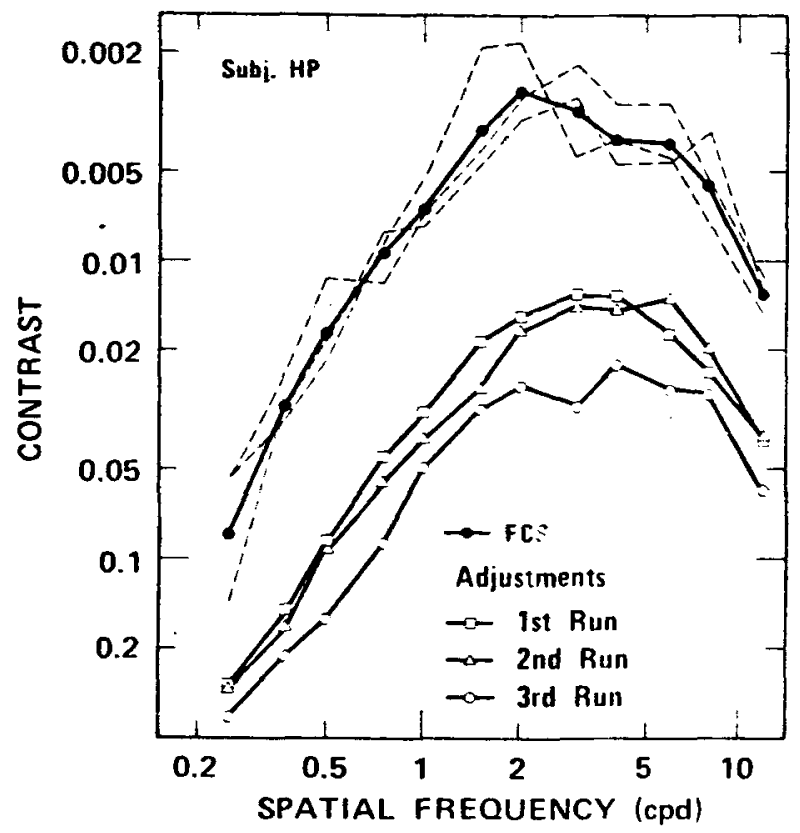

Fig. 5. Contrast sensitivity vs spatial frequency for S H.P. Same conditions as Figs. 3 and 4. Dashed lines show data from three FCS runs, averaged to obtain filled-circle points.

times greater than the adjustment sensitivity.

The most important result shown in Fig. 5 is the nature of the variability among FCS runs and among adjustments runs. Although the FCS data are about as variable as the adjustment data, the adjustment variability is mainly systematic, while the variability among the FCS runs seems random. (Note that the FCS curves from individual sessions cross and recross each other and the mean curve several times.) The data were subjected to a chi-square test of the hypothesis that mean rank contrast sensitivity is independent of session number. This hypothesis can be rejected for the adjustments data $(\mathrm{p}<.001)$, but cannot be rejected for the FCS data $(p=.1)$.

Now, if the FCS method eliminates systematic intra-S variability from one run to the next, and if this intersession variability is caused mainly by changes in S's criterion, then we would expect this method to minimize the variability among $S s$ as well. The data shown in Fig. 6 tend to confirm this expectation. Here the mean FCS curve for S H.P. is repeated, together with similar data for two other young, emmetropic Ss. Note the close similarity among all three contrast sensitivity curves, particularly at frequencies below $2 \mathrm{cpd}$. These data (and others not reported here) all tend to confirm the presence of a low-frequency inhibiting effect with a relatively steep slope (about 2 in $\log \cdot \log$ coordinates).

\section{DISCUSSION}

Instructing $S$ to try to detect any perturbation of the uniform field may be important in making the adjustment curves so similar in shape to the FCS curves. One should not assume that our results would be obtained if $S$ were instructed to detect the presence of a grating, or to report its orientation, count its fringes, etc. The use of other subjective criteria can doubtless change the shape of the sine wave threshold curve.

When minimum threshold values are not required, and occasional criterion differences among and within Ss can be tolerated, the method of adjustments is obviously preferable, because it yields equally smooth curves in about a 10th of the time required by the FCS method. ${ }^{1}$ However, the FCS method eliminates criterion differences and provides information about S's performance (not just his judgments).

Summarizing our results with five Ss, ranging from naive to quite experienced: (1) Both methods yield the same curve shape, but the FCS method gives significantly greater sensitivity than that obtained by the method of adjustments; the increase may be as great as a factor of 5 (depending on S's adjustment criterion). (2) Presumably because it is independent of threshold criteria, the FCS method does not show systematic changes of sensitivity from one run to the next (as the method of adjustments sometimes does.) (3) The variability of the FCS data is mainly random and can therefore be made quite small by taking enough data. (4) When this is done, individual differences (among young, emmetropic Ss) tend to disappear.

These results are essentially what would be expected, based on the differences between the two psychophysical methods. We conclude that, if the $\mathrm{S}$ is appropriately instructed, the shape of his sine wave grating sensitivity curve is not affected by using the method of adjustments. Moreover, it seems likely that this negative result would be maintained if the present study were extended to other criterion-dependent

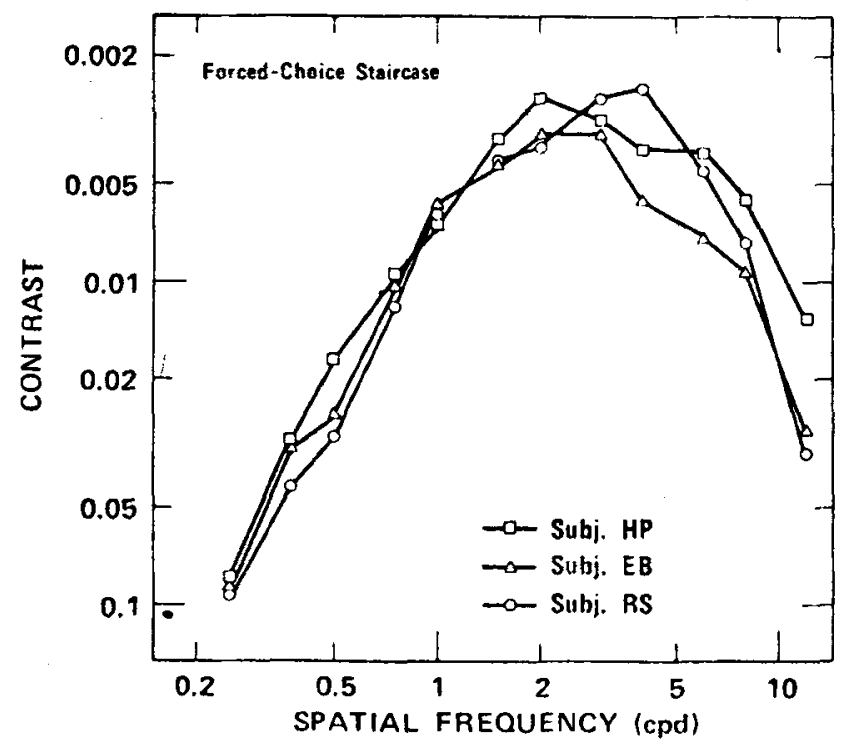

Fig. 6. Contrast sensitivity vs spatial frequency for three young, emmetropic Ss, obtained by the FCS technique. 


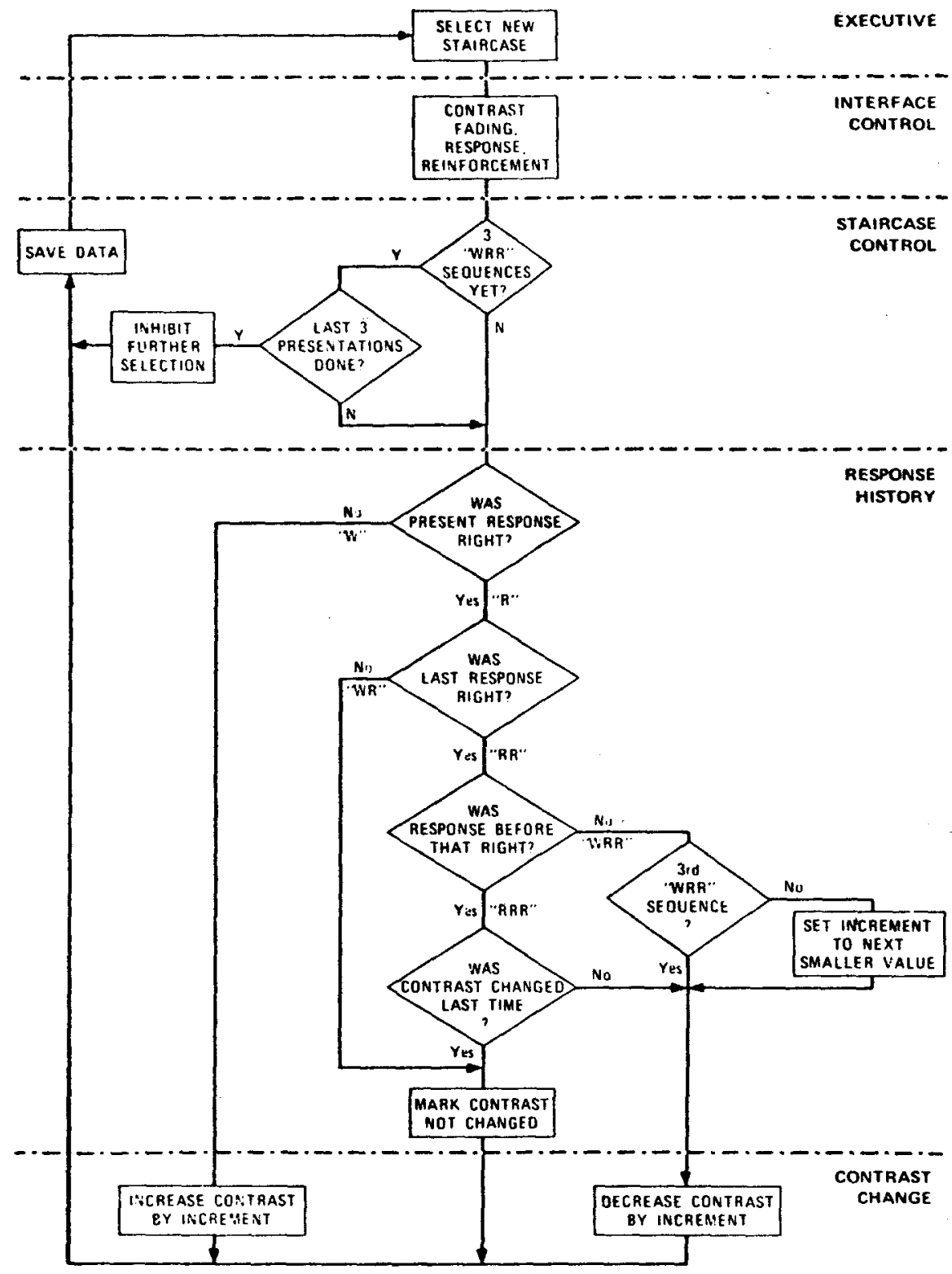

Fig. 7. Flow-chart representation of the FCS computer program.

psychophysical methods in general. Thus, our results tend to support the sine wave threshold data obtained by these methods.

\section{APPENDIX}

\section{The Forced-Choice Staircase (FCS) Computer Program}

The operation of the FCS computer program can be divided into five functional categories: executive, interface control, staircase control, response history, and contrast change (see Fig. 7).

The executive section of the program initializes the experiment, selects (from a random table) one of the 12 stimulus patterns for presentation at each trial, performs various bookkeeping functions, and calculates and prints out the average data at the end of an experimental session. The staircase control portion of the program operates on a parameter table for the staircase currently selected; it keeps track of the previous three responses, the previous eight contrasts, the present contrast, and contrast increment size (and other parameters for internal use). These historical data are needed for each staircase to determine its subsequent contrasts.

The interface control portion of the program controls the stimulator interface, which operates the entire sequence of each trial: stimulus generation, contrast fading, marker tones, and reinforcement. When $\mathrm{S}$ makes a response, the computer reads data from the interface, indicating whether the response was right or wrong (or 
whether the "pause" switch was set).

The response history section is the heart of the FCS program; Fig. 7 shows a flow chart of this section and its relation to the other parts of the program. Each staircase follows the algorithm according to its own history, independent of the other staircases. The next contrast to be presented (for the selected staircase) is calculated on the basis of the present contrast, the present response, the previous two responses, the contrast increment, and whether the contrast was changed after the previous response. The contrast algorithm is as follows: (1) When $S$ makes a wrong (W) response, the contrast of that pattern is increased for its next presentation. After he makes two successive right ( $R$ ) responses to the same pattern, its contrast is decreased (this sets the average end point of each staircase at about $75 \%$ correct). The new contrast is obtained by multiplying (or dividing) the preceding contrast by a fixed ratio (i.e., a constant $l o g$ increment). (2) Each time the sequence W, R, R occurs, the size of this increment is decreased. (This is most likely to occur when the contrast increases from below to above the $75 \%$ correct level.) The successive increment sizes are: $8 X, 2 \mathrm{X}, 1.3 \mathrm{X}$. (3) After the seguence $W, R, R$ has occurred three times with a given pattern, the staircase terminates with three more trials using the smallest increment, and that pattern is not presented again. The session ends when all staircases have terminated.

The last eight contrasts from each staircase are averaged to obtain the threshold. The minimum number of trials before termination but after the second $W, R, R$ sequence is eight. Therefore, all contrasts being averaged were necessarily presented during the period when the smallest contrast increment was in effect.

\section{REFERENCES}

Békésy, G. v. A new audiometer. Acta Oto-laryngologica, 1947, $35,411-422$.

Blackwell, H. R. Contrast thresholds of the human eye. Journal of the Optical Society of America, 1946, 36, 624-643.

Campbell, F. W., \& Green, D. G. Optical and retinal factors affecting visual resolution. Journal of Physiology, 1965, 181, 576-593.

Cornsweet, T. N. The staircase method in psychophysics. A merican Journal of Pychology, 1963, 75, 483-491.

Davidson, M. A perturbation analysis of spatial brightness interaction in flashed visual fields. Doctoral thesis, University of California, Berkeley, 1966.

Heinemann, E. G. The relation of apparent brightness to the threshold for differences in luminance. Journal of Experimental Psychology, 1961, 61, 389-399.
Kelly, D. H. F requency doubling in visual responses. Journal of the Optical Society of A merica, 1966, 56, 1628-1633.

Kelly, D. H. Effects of sharp edges on the visibility of sinusoidal gratings. Journal of the Optical Society of America, 1970, 60 . 98-103.

Kelly, D. H. Transient effects on the visibility of low spatial frequencies. Journal of the Optical Society of America, 1971, $61,1576(\mathrm{~A})$.

Kelly, D. H. Adaptation effects on spatio-temporal sine-wave thresholds. Vision Research, 1972, 12, 89-101.

Kelly, D. H. Lateral inhibition in human colour mechanisms. Journal of Physiology, 1973, 228, 55-72.

Kohayakawa, Y. Contrast-difference thresholds with sinusoidal gratings. Journal of the Optical Society of America, 1972, 62, 584-587.

Rose, R. M., Teller, D. Y., \& Rendleman, P. Statistical properties of staircase estimates. Perception \& Psychophysics, 1970, 8, 199-204.

Van Meeteren, A. Spatial sinewaye response of the visual system (a critical literature survey). Soesterberg: Institute for Perception RVO-TNO, 1966.

Westheimer, G. Modulation thresholds for sinusoidal light distributions on the retina. Journal of Physiology, 1960, 152, 67-74.

\section{NOTE}

1. Because the FCS sessions are long and tiresome, a number of preliminary experiments were conducted to determine the most suitable compromise between speed and end-point stability. We found that the variability shown in Fig. 5 could not be decreased significantly without prohibitive increases in run length. The number and sizes of our increments were governed by the following constraints (see Cornsweet, 1963): (a) For maximum efficiency, the smallest increment must be fixed at about the same size as the contrast-difference threshold for sinusoidal gratings; at a spatial frequency of 2.1 cycles/deg, Kohayakawa (1972) obtained jnds of $30 \%$ to $60 \%$ with low-contrast gratings. (Note that this is much larger than the jnd of luminance.) (b) All staircases must start at a common baseline; for this purpose, we chose $100 \%$ contrast. (To start at equal distances from threshold would require an a priori assumption of the curve shape we are measuring, which could bias the result.) (c) If the contrast is to approach threshold quickly, the initial increment must be much larger than specified in (a). The increment should decrease as the contrast approaches threshold, but not so rapidly as to run a high risk of being "trapped" in a small increment far from threshold. (d) Subject to constraint (c), the total number of increments should be minimized.

(Received for publication January 16, 1973; accepted March 15, 1973; revision received March 29, 1973.) 